\title{
Learning Real-World Stimuli by Single-Spike Coding and Tempotron Rule
}

\author{
Huajin Tang \\ Institute for Infocomm Research \\ Agency for Science Technology and \\ Research (A*STAR) \\ Singapore 138632 \\ Email: htang@i2r.a-star.edu.sg
}

\author{
Qiang Yu \\ Department of Electrical and \\ Computer Engineering \\ National University of Singapore \\ Singapore 117576 \\ Email: yu.qiang@nus.edu.sg
}

\author{
K.C. Tan \\ Department of Electrical and \\ Computer Engineering \\ National University of Singapore \\ Singapore 117576 \\ Email: eletankc@nus.edu.sg
}

\begin{abstract}
In this paper, a system model is built for pattern recognition by using spiking neurons. The system contains encoding, learning and readout. The schemes used in this network are efficient and biologically plausible. Through the encoding of our network, the external stimuli (images) are converted into spatiotemporal spiking patterns. These spiking patterns are then efficiently learned through a supervised temporal learning rule. Through simulation, the properties of the system model are shown. It turns out that this network can successfully recognize different patterns very fast.
\end{abstract}

\section{INTRODUCTION}

Pattern recognition has been performed by many conventional methods, like maximum entropy classifier, naive Bayes classifier, decision trees, support vector machines and perceptrons. We refer these methods as conventional ones since most of them lack biological plausibility. Comparing to these conventional methods, the biological counterpart (like human brain) can perform recognition task better both in accuracy and speed. This motivates us to consider a system model for pattern recognition which is more biologically plausible.

We construct the general structure of our system model as feed-forward one which contains 3 functional parts: the encoding part, the learning part and the readout part (see Fig. 1). The encoding one generates a set of specific activity patterns used for learning neurons. These patterns represent the various attributes of external stimuli. The learning neurons learn these patterns by modifying their synaptic weights through corresponding rule and store these synaptic weights as memories. The final decision is presented in the readout.

The first step to recognize patterns is to understand the way how information is stored in the pattern. It is still unclear how the information is represented in the brain. However, there are strong evidences showing that the representation of external stimuli in the brain is in a form of spatiotemporal spikes [1], [2]. How these spikes encode information in the brain still remains unclear. Two of the widely studied coding methods are rate coding and temporal coding [3]. Rate coding is the most basic example of a neural code in which information is conveyed through spike count within an encoding window [3]. In rate coding, neurons' mean firing rates are related to sensory variables (this is demonstrated in [4]). However,

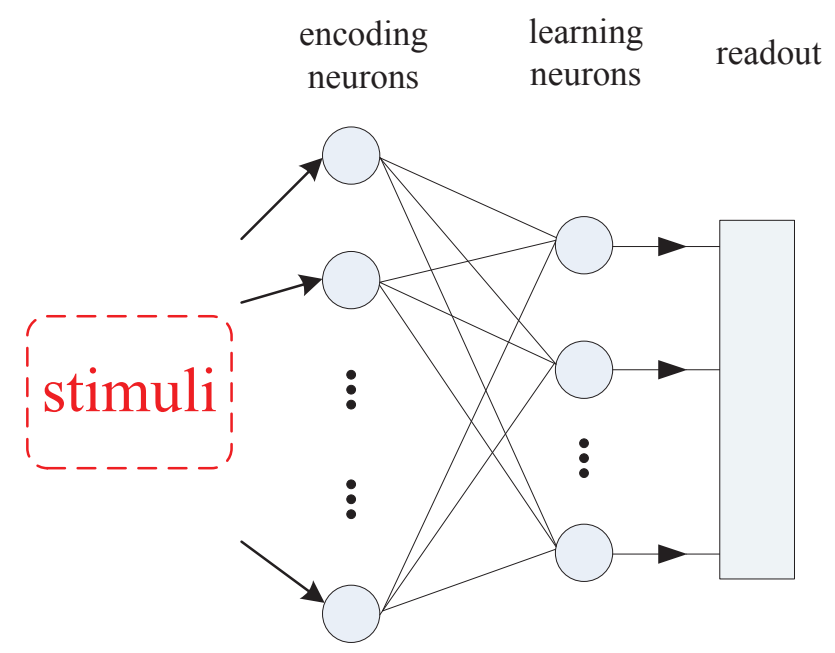

Fig. 1. The general structure of the brain based model for pattern recognition. It contains three parts: encoding, learning and readout. A stimulus is firstly presented to the encoding part and is converted into a proper pattern which is used for the following learning neurons. The learning neurons store these patterns according to the learning rule and the final decision is presented in the readout.

many experimental observations, such as in the retina [5], [6], the lateral geniculate nucleus (LGN) [7] and the visual cortex [8], [9], shows that neurons precisely respond to stimuli on a millisecond timescale. These observations support the hypothesis of temporal coding in which precise timings of spikes are considered for conveying information. Recent studies have shown that temporal coding patterns can carry more information than rate coding patterns [10]-[12]. There are several examples of temporal coding that have been proposed, such as interspike intervals coding and phase of firing coding [3], latency coding [13], temporal order coding [14]. The primate visual system can analyze a new complex scene in less than $150 \mathrm{~ms}$ [15], [16]. This period of time is impressive for information processing considering billions of neurons involved. This suggests that neurons exchange only one or few spikes. Thus, we use single spike coding as the encoding mechanism which is simple and efficient. Our encoding is 
similar to the rank order coding in [14], [17], but considering the precise latency of the spikes which could convey more information.

Several brain-like neural models, which are biologically plausible, have been proposed, such as the leaky integrateand-fire (LIF) model [18], the Izhikevich model [19] and the Hodgkin-Huxley-type model [20]. The LIF model, to some extent, is simple and computationally effective [21]. Thus, we choose the LIF model to construct our network. In addition, we choose a biologically plausible supervised learning rule, the tempotron rule [22], to direct how the neurons modify their synaptic weights. This learning rule has been demonstrated to be able to classify a large number of spatiotemporal patterns.

We describe a system model based on biological evidences for pattern recognition. The system model contains several functional parts: encoding, learning and readout. Our system model could be used in pattern recognition task for realworld stimuli (images). Through simulation, we show that our network could classify a large number of patterns successfully.

\section{TEMPORAL CODING}

Our encoding layer is used to convert stimulus into spatiotemporal spikes using single spikes. In this section, we describe how the encoding model is built to mimic the performance of biological processing in retina.

The general structure and functional organization of the retina are well known. It is widely believed that information transmitted from retina to brain codes the intensity of the visual stimuli at every place in visual field. The ganglion cell (GC) collects the information from its receptive fields which could best drive spiking responses [23]. In addition, different ganglion cells might have overlapped centers of receptive fields [24]. The encoding model is shown in Fig. 2. As in [17], the GCs collect the image information (intensity) through corresponding photo-receptors in their receptive field. The receptive fields of GCs are overlapping and are generally distributed non-uniformly over the visual field. The activation value of a GC could be computed as the following equation:

$$
C_{i}:=<I, \phi_{i}>=\sum_{l \in R_{i}} I(l) \cdot \phi_{i}(l)
$$

where $I(l)$ is the luminance of pixel $l$ which is sensed by the photo-receptor. $R_{i}$ is the receptive field region of neuron $i . \phi_{i}$ is the weight of the filter.

The GCs perform like analog-to-delay converters. The ones that are strongly activated will fire earlier, whereas the ones that are weakly activated will fire later or not at all. We use the simple DoG [25] as our filter where the surround has three times the width of the center:

$$
D o G_{\left\{s, l_{c}\right\}}(l)=G_{\sigma(s)}\left(l-l_{c}\right)-G_{3 \cdot \sigma(s)}\left(l-l_{c}\right)
$$

where $G_{\sigma(s)}$ is the 2-D Gaussian function with variance $\sigma(s)$ which depends on the scale $s . l_{c}$ is the center position of the filter.

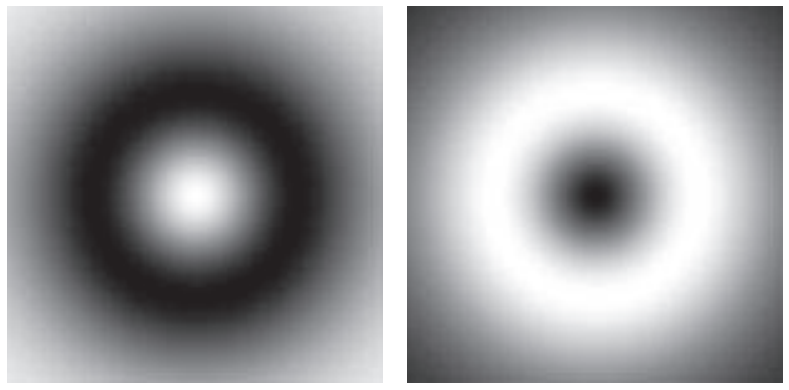

Fig. 3. Illustration of DoG filters. The left is an image of the Center-ON filter; the right is an image of the Center-OFF filter.

The Gcs compute the local contrast intensities at different spatial scales and for two different polarities: ON- and OFFcenter filters. Fig. 3 illustrates examples of the DoG filter. A center-OFF filter is simply an inverted version of a centerON receptive field. The activation values of GCs are used to produce spikes in which the spike latencies are inversely proportional to the activation of the neuron. The spike latencies are linearly mapped into an encoding time window.

Through the encoding model, the input images can be converted into spatiotemporal spikes which are used for further computation in following layers. Each GC refers to a particular part of the image. The information of this part is encoded in the spike emitted from the GC.

\section{Temporal LEARning Rule}

In this section, we describe the learning rule we used to deal with information encoded in precise timing spikes. Among different temporal learning rules, one of the most commonly and experimentally studied rules is spike-timingdependent plasticity (STDP). In STDP, the basic mechanisms of plasticity are long term potentiation (LTP) and long term depression (LTD). LTP occurs when presynaptic spike precedes postsynaptic one; LTD occurs when postsynaptic spike precedes presynaptic one (see Fig. 4). Being compatible with STDP rule, the tempotron rule is suitable for classifying a great number of spatiotemporal patterns [22]. In the tempotron rule, both LTP and LTD occur under the supervision of a teacher signal. The tempotron rule is a biologically plausible supervised learning rule which enables neurons to efficiently learn a broad range of patterns. In this paper, we use the tempotron rule as our learning rule.

According to the tempotron rule, the efficacies of afferent synapses are modified such that the trained neuron emits one spike presented to one category and no spike presented to the other category. The neuron model is a leaky integrateand-fire (LIF) neuron driven by exponential decaying synaptic currents generated by its afferent synapses. The subthreshold membrane voltage $U(t)$ of the neuron is a weighted sum of postsynaptic potentials (PSPs) from all incoming spikes:

$$
U(t)=\sum_{i} w_{i} \sum_{t_{i}} E\left(t-t_{i}\right)+U_{\text {rest }}
$$




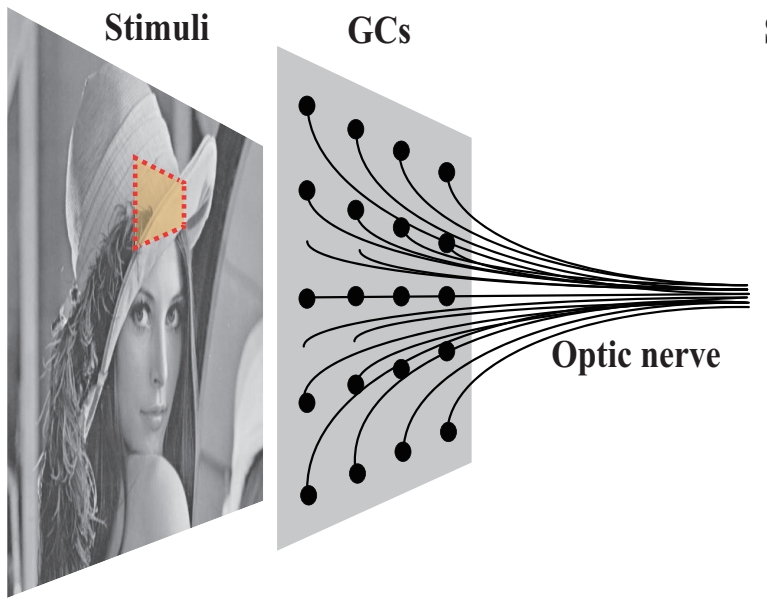

\section{Spatiotemporal spikes}

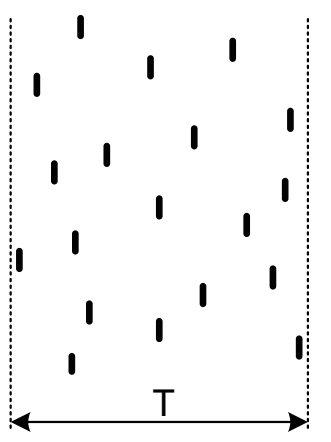

Fig. 2. Architecture of the retina model. A gray-scale image (as the stimuli) is presented to the encoding layer. The photo-receptors transmit the image information analogically and linearly to the corresponding ganglion cells (GCs). Each ganglion cell collects information from its receptive field (an example shown as the red dashed box), and fire a spike according to their activations. These spikes of all the GCs are transmitted to the next layer as the spatiotemporal pattern in a particular time window (T).

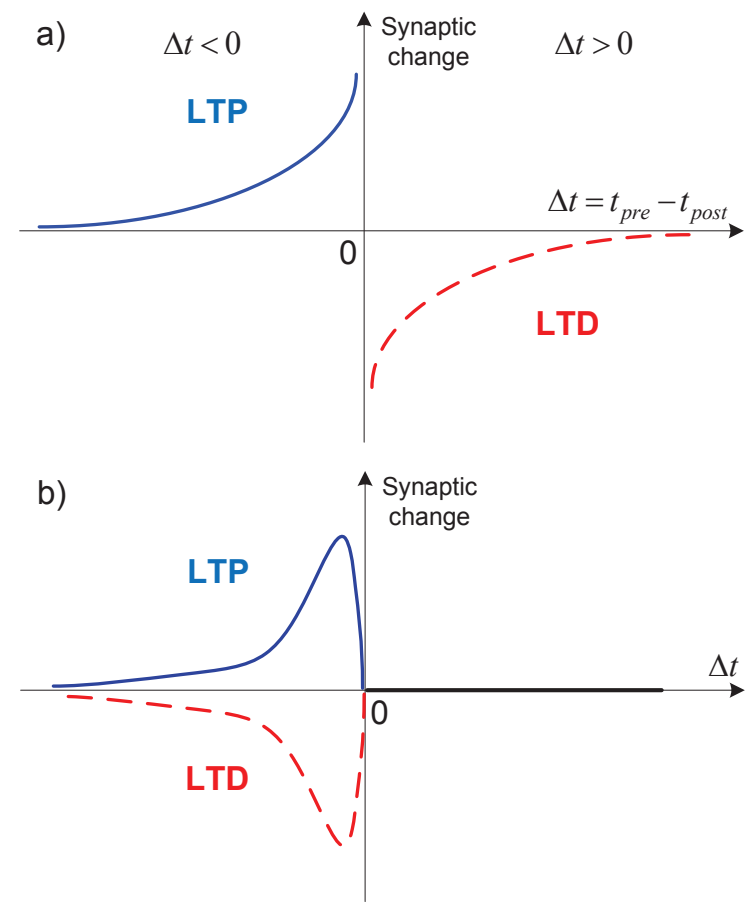

Fig. 4. Learning windows of different temporal rules. The blue lines denote the LTP process and the dashed red ones denote the LTD process. a. is the learning window for STDP; b. is for tempotron rule.

where $w_{i}$ and $t_{i}$ are the synaptic efficacy and the firing time of the $i^{t h}$ afferent. $U_{\text {rest }}$ is the rest potential of the neuron. $E$ denotes the normalized PSP kernel:

$$
E\left(t-t_{i}\right)=E_{0}\left(\exp \left(\frac{-\left(t-t_{i}\right)}{\tau_{m}}\right)-\exp \left(\frac{-\left(t-t_{i}\right)}{\tau_{s}}\right)\right)
$$

where $\tau_{m}$ and $\tau_{s}$ are decay time constants. $E_{0}$ normalizes PSP so that the maximum value of the kernel is 1 .

Before being presented with an input pattern, the neuron's potential is at rest. When $U(t)$ crosses the threshold, the neuron emits a spike, after which the potential gradually decreases to the rest value by shunting all the following input spikes. The dynamic is illustrated in Fig. 5.
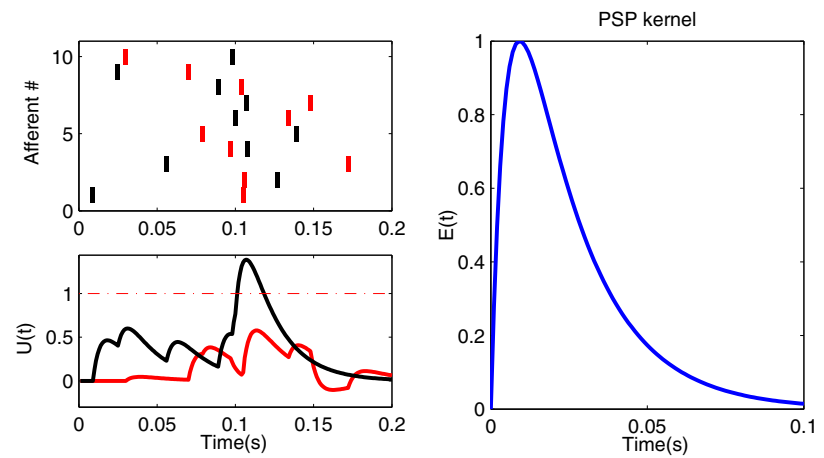

Fig. 5. Illustration of the neural respond. Left top: examples of spiking patterns. Two patterns (black and red) are shown. Each spike from an input afferent is denoted by a bar. Left bottom: neural potential traces. Each color of lines refers to corresponding colored patterns on left top. The firing threshold is 1. Right: normalized PSP kernel.

The neuron classifies different patterns under a supervisory signal. Supposing two categories of patterns $\left(\mathrm{P}^{+}\right.$and $\left.\mathrm{P}^{-}\right)$ to be classified, the neuron should emit a spike when it is presented to a $P^{+}$pattern and keep silent when it is presented to a $P^{-}$pattern. This rule minimizes the following cost function:

$$
C= \begin{cases}U_{t h r}-U_{t_{\max }}, & \text { if the presented pattern is } P^{+} \\ U_{t_{\max }}-U_{t h r}, & \text { if the presented pattern is } P^{-}\end{cases}
$$

where $U_{t_{\max }}$ is the maximal value of the postsynaptic potential $U(t)$.

Applying the gradient descent method to minimize the cost leads to the tempotron learning rule: 


$$
\Delta w_{i}=\left\{\begin{array}{lc}
\lambda \sum_{t_{i}<t_{\max }} E\left(t_{\max }-t_{i}\right), & \text { if } P^{+} \text {error; } \\
-\lambda \sum_{t_{i}<t_{\max }} E\left(t_{\max }-t_{i}\right), & \text { if } P^{-} \text {error; } \\
0, & \text { otherwise. }
\end{array}\right.
$$

where $t_{\max }$ denotes the time at which the neuron reaches its maximum potential value. $\lambda>0$ is a positive constant as the learning rate. $P^{+}$error means that the neuron should fire but it did not; $P^{-}$error means that the neuron should not fire but it did.

In this learning rule, LTP is activated if the neuron failed to spike on a $P^{+}$pattern and LTD is activated if the neuron erroneously fired a spike on a $P^{-}$pattern.

\section{Simulation Results}

In this section, we perform the system model on the realworld stimuli (images). The stimuli from real world typically have a complex statistical structure which is different from idealized case of random patterns often considered. In real world, stimuli hold variability within a given class and correlation between different categories. The data set we considered here is the MNIST digits (see Fig. 6). All data in this set are gray-scale and on a grid of $28 \times 28$ pixels. The MNIST data set provides a good benchmark for our network performance. (The MNIST set is available from http://yann.lecun.com/exdb/mnist.)

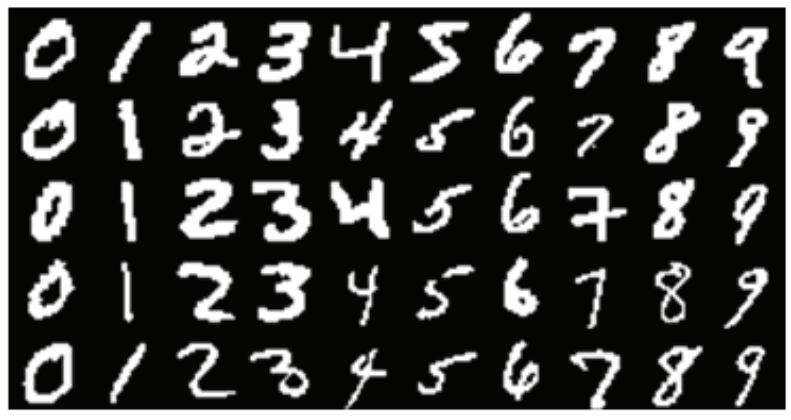

Fig. 6. Examples of data set. Handwritten digits from MNIST database

The data base provides a large number of samples for researching studies. As can be seen from Fig. 6, samples written by different people both differ from each other in the same category and overlap with others in different categories. A successful classification should be obtained through finding the differences among different categories and the similarities among the same category.

\section{A. Encoding images}

The encoding is a process by which information from the real-world stimuli (images) is converted into another representation (spikes). It is a key component in the system, because a good encoding method can hold adequate information of the original stimuli, and also can facilitate the later learning process.

Images are presented to the encoding layer where the retina model is used for encoding. For simplicity, we distribute all the GCs uniformly over the image. We choose two scales for our filters $(\sigma=0.5$ for $3 \times 3$ pixels as scale 1 , and $\sigma=1$ for $6 \times 6$ pixels as scale 2$)$.

Each GC gets an activation value $\left|C_{i}\right|$ by computing information in their receptive fields. The GC emits a spike in which the latency is based on $\left|C_{i}\right|$. The strongly activated cell will fire earlier, whereas the weakly activated will fire later or not at all. The latencies of spikes are inversely proportional to $\left|C_{i}\right|$, and then are linearly mapped into a predefined time window (we choose $256 \mathrm{~ms}$ here). Fig. 7 shows the encoding example. Those of non-fired spikes, which corresponding to lower activation values, are aligned at the end of encoding window for illustration. These spikes are not involved in the computation of spiking neurons.

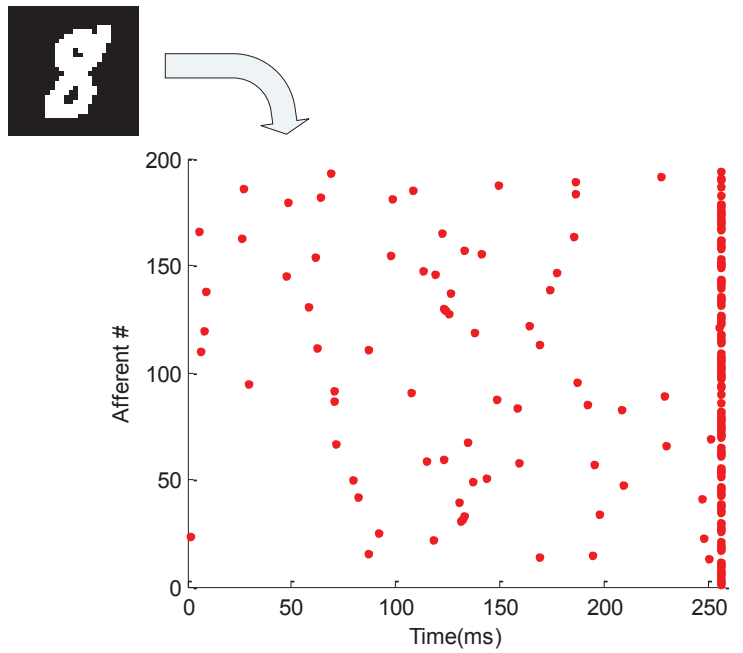

Fig. 7. Illustration of the performance of encoding layer. The encoded image ("8") is chosen from MNIST. The length of the encoding window is $256 \mathrm{~ms}$. The total number of the afferent inputs is 194. Each dot denotes a spike. Strong activated neuron will fire early. Those neurons that not activated enough would not fire at all. For illustration of those non-fired spikes, they are aligned at the end of time window.

As can be seen from Fig. 7, the spikes conveying relatively important information are distributed sparsely in the encoding window. The spareness of our encoding is compatible with biological observations in retinas [26]. In addition, most of spikes corresponding to the background is placed at the end of the encoding window. This could benefit an efficient information processing since the background information is relatively unimportant.

The spatiotemporal spiking pattern is composed of single spikes which could benefit the computational speed. In addition, highlighting the relatively important information, by putting corresponding spikes at the beginning of the encoding window, could also result in an efficient computation.

\section{B. Recognition Results}

To see the performance ability of this method on the recognition task in the network, we use a small data set from the MNIST (50 digits and 5 for each category). We set the ratio between the membrane and the synaptic constants to be 
$\tau_{m} / \tau_{s}=4 . U_{t h r}=1$ and $U_{\text {rest }}=0$ are chosen. We use $\tau_{m}=20 \mathrm{~ms}$ and $\lambda=0.002$. We choose 10 neurons as our readout and each neuron corresponds to one category.

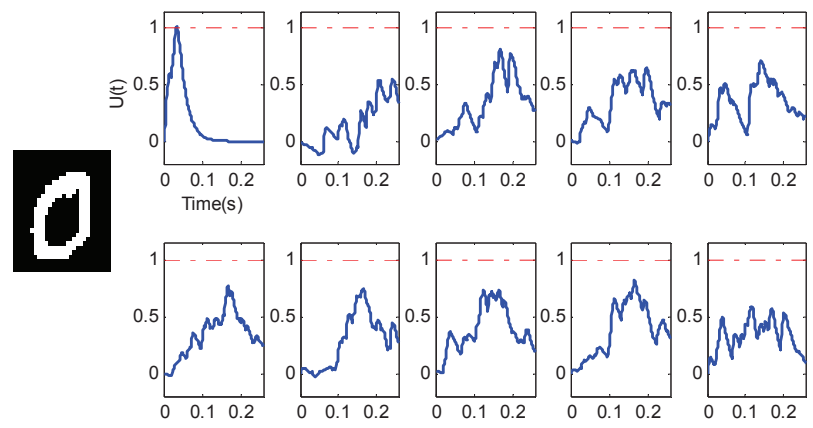

Fig. 8. Recognition results of digits by Tempotron learning rule. Here shows 10 learning neurons (Neuron 0 to 9) and 1 image ("0"). The neuron responds to an image by firing (1) or not (0). When an image is presented to the network, the corresponding neuron fires while others keep silent. For image " 0 ", only neuron 0 emits a spike.

As can be seen from Fig. 8, the neurons can classify different categories successfully by making a corresponding respond after several training times. When an image is presented to the network, the corresponding neuron fires while others keep silent. For example, if image " 0 " is presented, only neuron 0 emits a spike. Through modifying the weights, a neuron could successfully respond to the inputting stimuli. It would emit a spike on all patterns in the same corresponding category since the similarity in this category is found; it would keep silent on patterns in other categories since the difference between the corresponding category and others is found.

\section{Learning Speed}

To illustrate the learning speed of our network, we perform the task on the same data set as before with the 50 images. We perform it 30 times and a different initial condition (like the weights) is chosen for each time. The learning speed is illustrated in Fig. 9.

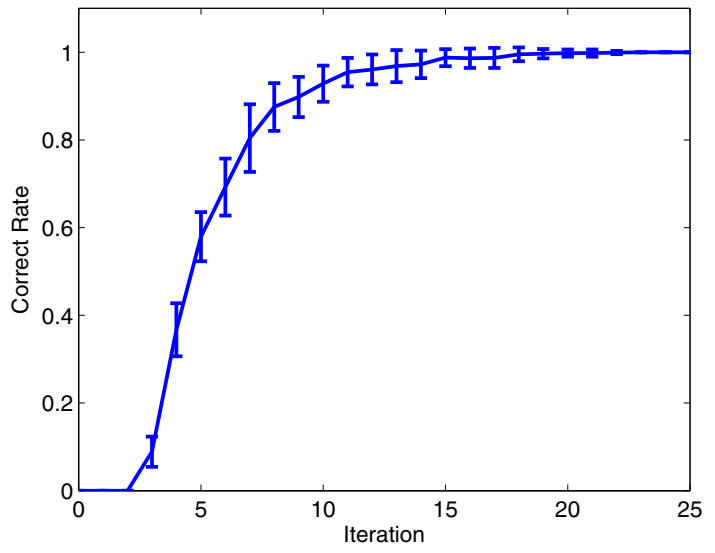

Fig. 9. Learning speed. The classification task is performed on a set of 50 images for 30 times. In each time, a different initial condition is chosen.
As can be seen from Fig. 9, the recognition task could be successfully done in tens of iterations. At the first several iterations, the learning is very fast. The correct rate of recognition can reach to a relatively high value in several steps. This implies that the network could easily discriminate a large number of images from different categories which are very different. At the last several iterations, the learning slows down. There is no significant improvement on the correct rate of recognition. This implies the network is trying to discriminate similar images. Since images from different categories overlap with each other, it is reasonable for slowing down the learning to classify the patterns.

\section{CONCLUSION}

We have built a system model using spiking neurons for pattern recognition. The system contains encoding, learning and readout. The schemes used in this network are efficient and biologically plausible. Through our network, the temporal learning rule used in this paper can be applied to process realworld stimuli (images). Through simulation, the properties of this model are shown. It turns out that the network can successfully recognize different categories very fast.

In addition, the tempotron rule has also been proved quite efficient for spoken digit recognition [27], having comparable performance applied in spiking neural networks to that achieved by complex and biologically implausible state-ofthe-art speech-recognition systems such as the Hidden Markov model and HTK. These show the spiking neural networks are quite powerful in kinds of recognition tasks. One limitation of the tempotron learning rule is the need to compute the time of the maximum of the postsynaptic voltage. This could lead to a further research on appropriate learning methods for training the spiking neural networks. These learning methods might reveal even more potential of the spiking neurons.

\section{ACKNOWLEDGMENT}

This work was supported by Agency for Science, Technology, and Research (A*STAR), Singapore under SERC Grant 0921570130 .

\section{REFERENCES}

[1] F. Rieke, D. Warland, Rob, and W. Bialek, Spikes: Exploring the Neural Code, 1st ed. Cambridge, MA: MIT Press, 1997.

[2] O. Barak and M. Tsodyks, "Recognition by variance: Learning rules for spatiotemporal patterns," Neural Comput., vol. 18, pp. 2343-2358, October 2006.

[3] S. Panzeri, N. Brunel, N. K. Logothetis, and C. Kayser, "Sensory neural codes using multiplexed temporal scales," Trends in Neurosciences, vol. 33, no. 3, pp. 111-120, Mar. 2010.

[4] E. Adrian, The Basis of Sensation: The Action of the Sense Organs. W. W. Norton, New York, 1928.

[5] M. J. Berry and M. Meister, "Refractoriness and neural precision." $J$ Neurosci, vol. 18, no. 6, pp. 2200-2211, Mar. 1998.

[6] V. J. Uzzell and E. J. Chichilnisky, "Precision of spike trains in primate retinal ganglion cells." J Neurophysiol, vol. 92, no. 2, pp. 780-789, Aug. 2004.

[7] P. Reinagel and R. C. Reid, "Temporal coding of visual information in the thalamus," Journal of Neuroscience, vol. 20, no. 14, pp. 5392-5400, Jul. 2000. 
[8] W. Bair and C. Koch, "Temporal precision of spike trains in extrastriate cortex of the behaving macaque monkey." Neural Comput, vol. 8, no. 6, pp. 1185-1202, Aug. 1996.

[9] Z. F. Mainen and T. J. Sejnowski, "Reliability of spike timing in neocortical neurons," Science, vol. 268, no. 5216, pp. 1503-1506, Jun. 1995.

[10] R. Kempter, W. Gerstner, and J. L. van Hemmen, "Spike-based compared to rate-based hebbian learning." in NIPS'98, 1998, pp. 125-131.

[11] A. Borst and F. E. Theunissen, "Information theory and neural coding." Nature neuroscience, vol. 2, no. 11, pp. 947-957, Nov. 1999.

[12] J. J. Hopfield, "Pattern recognition computation using action potential timing for stimulus representation," Nature, vol. 376, no. 6535, pp. 33-36, Jul. 1995.

[13] T. J. Gawne, T. W. Kjaer, and B. J. Richmond, "Latency: another potential code for feature binding in striate cortex," $J$ Neurophysiol, vol. 76, no. 2, pp. 1356-1360, Aug. 1996.

[14] R. Van Rullen and S. J. Thorpe, "Rate coding versus temporal order coding: what the retinal ganglion cells tell the visual cortex." Neural Comput, vol. 13, no. 6, pp. 1255-1283, Jun. 2001.

[15] S. Thorpe, D. Fize, and C. Marlot, "Speed of processing in the human visual system," Nature, vol. 381, no. 6582, pp. 520-522, Jun. 1996.

[16] T. Gollisch and M. Meister, "Rapid neural coding in the retina with relative spike latencies." Science, vol. 319, no. 5866, pp. 1108-1111, 2008.

[17] L. Perrinet, M. Samuelides, and S. J. Thorpe, "Coding static natural images using spiking event times: do neurons cooperate?" IEEE Transactions on Neural Networks, vol. 15, no. 5, pp. 1164-1175, 2004.

[18] W. Gerstner and W. M. Kistler, Spiking Neuron Models: Single Neurons, Populations, Plasticity, 1st ed. Cambridge University Press, Aug. 2002.

[19] E. M. Izhikevich, "Simple model of spiking neurons," IEEE Transactions on Neural Networks, vol. 14, no. 6, pp. 1569-1572, Nov. 2003.

[20] A. L. Hodgkin and A. F. Huxley, "A quantitative description of membrane current and its application to conduction and excitation in nerve." The Journal of physiology, vol. 117, no. 4, pp. 500-544, Aug. 1952.

[21] E. M. Izhikevich, "Which model to use for cortical spiking neurons?" IEEE Transactions on Neural Networks, vol. 15, no. 5, pp. 1063-1070, September 2004.

[22] R. Gütig and H. Sompolinsky, "The tempotron: a neuron that learns spike timing-based decisions," Nature Neuroscience, vol. 9, no. 3, pp. 420-428, Feb. 2006.

[23] D. H. Hubel and T. N. Wiesel, "Receptive fields and functional architecture of monkey striate cortex." The Journal of physiology, vol. 195, no. 1, pp. 215-243, Mar. 1968.

[24] Burkart and Fischer, "Overlap of receptive field centers and representation of the visual field in the cat's optic tract," Vision Research, vol. 13, no. 11 , pp. 2113 - 2120, 1973.

[25] D. J. Field, "What is the goal of sensory coding?" Neural Comput., vol. 6, pp. 559-601, July 1994.

[26] B. A. Olshausen and D. J. Field, "Sparse coding with an overcomplete basis set: a strategy employed by V1?" Vision research, vol. 37, no. 23 , pp. 3311-3325, Dec. 1997.

[27] R. Gütig and H. Sompolinsky, "Time-warp-invariant neuronal processing." PLoS biology, vol. 7, no. 7, pp. e1 000 141+, Jul. 2009. 